\title{
Influence of Ar Gas Pressure On The Structural And Optical Properties And Surface Topography of Al- Doped ZnO Thin Films Sputtered By DC-Magnetron Sputtering Method
}

\author{
Mahdiyeh Shiravand \\ University of Malayer \\ Nader Ghobadi ( $\nabla$ n.ghobadi@malayeru.ac.ir) \\ University of Malayer https://orcid.org/0000-0002-9957-9395 \\ Ebrahim Gholami Hatam \\ University of Malayer
}

\section{Research Article}

Keywords: AL doped ZnO, Transition index, Urbach tail, Refractive index, dielectric constant, dissipation factor, (DITM), the strength of electron-phonon interaction, the lattice dielectric constant.

Posted Date: September 14th, 2021

DOI: https://doi.org/10.21203/rs.3.rs-691578/v1

License: (c) (1) This work is licensed under a Creative Commons Attribution 4.0 International License.

Read Full License 


\title{
Influence of Ar gas pressure on the structural and optical properties and surface topography of Al-doped $\mathrm{ZnO}$ thin films sputtered by DC-Magnetron sputtering method
}

\author{
Mahdiyeh Shiravand ${ }^{1}$, Nader Ghobadi*1 ${ }^{1}$ and Ebrahim Gholami Hatam ${ }^{1}$ \\ ${ }^{1}$ Department of Physics, Faculty of Science, Malayer University, Malayer, Iran \\ *Corresponding author Email address: n.ghobadi@malayeru.ac.ir
}

\begin{abstract}
In this work, Aluminum doped Zinc oxide thin films were sputtered on glass substrate by the direct current (DC) magnetron sputtering method. The influence of Ar gas pressure on the structural and optical properties was measured. The optical parameters were calculated by UV-Visible spectroscopy, the nature of transition reveals direct allowed transition for the prepared films. Also, some physical quantities such as the strength of electron-phonon interaction, dissipation factor $(\tan \delta)$ in the visible region and the lattice dielectric constant were presented for these thin films. The AFM analysis extracts surface parameters of the AZO thin films that help us to investigate the surface analysis with numerical data. The band gap energy and transition index without any presumption about transition natural were calculated from Derivation Ineffective Thickness Method (DITM) and the reaction of Ar gas pressure plays an essential part in controlling the physical quantities of AZO thin films.
\end{abstract}

\section{Keywords:}

AL doped $\mathrm{ZnO}$; Transition index; Urbach tail; Refractive index; dielectric constant; dissipation factor; (DITM); the strength of electron-phonon interaction; the lattice dielectric constant.

\section{Introduction}

Common transparent conductive oxide (TCO) thin films have been widely used in the photovoltaic application, therefor are marvelous for investigating [1-7]. The Zinc oxide thin films has categorized in the II-VI compound semiconductors. The interesting characteristics of $\mathrm{ZnO}$ such as its large exciton binding energy of $60 \mathrm{meV}$, wide band gap have attracted much regard for studying, in addition to the well-known advantages of undoped $\mathrm{ZnO}$ films, due to its other features such as high resistivity, instability, and low conductivity, it is not preferred, also for reducing the resistivity and modifying the electrical characteristics, 
the $\mathrm{ZnO}$ film is doped by the elements of group III such as $\mathrm{Al}, \mathrm{Ba}, \mathrm{Ga}$, and $\mathrm{In}$, it is become conducting nature owing to some free electrons was incorporated into $\mathrm{ZnO}$ films that these materials have been widely used for some devices such as gas sensors, transparent thin film transistors, solar cells, displays, electrochromic devices, and photocatalysis, etc. [2, 8- 16]. Among these TCO materials, the Aluminum doped on $\mathrm{ZnO}$ ( $\mathrm{AZO}$ ) thin films semiconductors are reported because of its attractive characteristics such as low resistivity, high optical transparency in the visible region, low cost, high electrical conductivity, direct transition and strong absorbance, etc. [2, 10,17-23].

In order to obtain high-quality AZO films, a variety of the deposition techniques may be used such as DC-magnetron sputtering, chemical bath deposition (CBD), sol-gel technique and pulsed laser deposition, etc. $[5,7,9,10,12,18,24-28]$.

In this paper, the AZO thin films that were affected from the variation of Ar gas pressure were deposited by DC-magnetron sputtering technique. This method was employed owing to its flexibility and ability for deposit films under vacuum, stoichiometry and large-area deposition, etc. [29- 31], in this technique the physical characteristics of AZO films such as composition, thickness and crystal phase, can be affected by the various sputtering parameters such as argon gas pressure, time of sputtering, and different concentration, etc. In this work the variation of Ar gas pressure plays an essential part for researching of the structural and optical properties of AZO films, Also DITM is reported for determining of the optical band gap and the type of optical transitions in nanostructured semiconductors.

Numerical factors for sample surfaces as used in material surface drawings are the means of communication between fabrication and functional performance. These factors are not only used as a criterion for production and surface parameter description, but also particularly in the case of 3D factors to predict physical properties. This initial numerical parameter as Root-mean-square deviation $\left(S_{q}\right)$, Skewness of topography height distribution $\left(S_{s k}\right)$, Kurtosis of topography height distribution $\left(S_{k u}\right)$, and inclination angles determined from the AFM images help researchers to investigate the surface analysis with numerical data [32-34].

\section{Experimental details}

In this paper, AZO thin films were sputtered by DC-magnetron sputtering method. A disk of AZO (purity of $99.9 \%$ ) with a diameter of $100 \mathrm{~mm}$ was utilized as a cathode. For this study, all of films sputtered with the same condition including $\mathrm{I}=175 \mathrm{~mA}$ (incoming current) and V=300 v (incoming voltage).

Before a deposition, for removing any embedded particles on the surface of substrates, the glass substrates were completely cleaned in a bath of ultrasonic with acetone and ethyl alcohol for 18 minutes. Then they were rinsed in distilled water and dry in hot air before they were located into the chamber. The system of 
vacuum have two pumps, a mechanical rotary and the turbo pumps are used for low and high vacuum in the deposition chamber, respectively. The substrates putting up in the chamber, the chamber was vacuumed to a base pressure of $2.8 \times 10^{-5}$ mbar prior to deposition then the pressure was enhanced and high purity Argon gas (99.99\%) was introduced through mass flow controller. In this study the AZO films sputtered in various Ar gas pressure which it was changed from $1 \times 10^{-2}$ mbar to $3 \times 10^{-2}$ mbar.

The absorption and transmittance, XRD, FESEM and AFM measurements were evaluated with Ultra Violet Visible double beam spectrophotometer (PerkinElmer, Lambda 25-USA), diffractometer (Unisantis-XMD300, $\mathrm{Cu} K_{\alpha}$, Germany), (Tscan,Mira 1ll,Czech Republic) and (Bruker, Icon, USA), respectively.

\section{Results and discussion}

\section{3-1. Structural study}

\section{3-1-1. XRD analysis}

Mainly, different structural features of AZO thin films were evaluated by X-ray diffraction [3, 20, 35]. Fig 1 illustrates the patterns of XRD for AZO films sputtered on a glass substrate by various Ar gas pressure from $1 \times 10^{-2}$ mbar to $3 \times 10^{-2}$ mbar [4, 12]. The patterns of XRD reveal only the same strong diffraction peak of (002) plane at $2 \theta$ location of $34.4^{\circ}$ that are very near to the wurtzite hexagonal $\mathrm{ZnO}$ crystal (34.43), also no $\mathrm{Al}_{2} \mathrm{O}_{3}$ phase was detected, this may be because of atoms of Al replace into the hexagonal lattice of Zinc or atoms of Al separated to the non-crystalline region in the grain boundary. [8, $16,17,19,23,36-38]$.

To obtain the detailed structural data, the mean crystallite size for (002) plane (the highest intensity peak) was calculated from the Debye Scherrer equation $[5,7,8,17,20]$.

$D=\frac{\mathrm{k} \cdot \lambda}{\beta \cos \theta}$

Where $\mathrm{k}$ is the particle-shape factor (0.9), $\lambda$ stands for the X-ray wavelength (K-Alpha 1: $1.5405(\AA), \beta$ is the line broadening at half the maximum intensity (FWHM) and $\theta$ is the Bragg angle of the diffraction peak with a variation of Ar gas pressure from $1 \times 10^{-2}$ mbar to $3 \times 10^{-2} \mathrm{mbar}$, in this study with enhancing Ar gas pressure, the mean crystallite size that indicate the longitude of the crystal in the orientation of the d-spacing increased from 10.2 - $19.9 \mathrm{~nm}$ and the FWHM of (002) peak decreased from 0.8160 to 0.4171 with enhancing Ar gas pressure which means the peak become more intense and sharper up to $3 \times$ $10^{-2}$ mbar, reducing of FWHM indicates the crystallinity of the AZO thin films is improved and the mean 
crystallite size becomes more extensive as the Ar gas pressure increased ( See Fig 2), the calculated results are listed in Table $1[7,18,26,29]$.

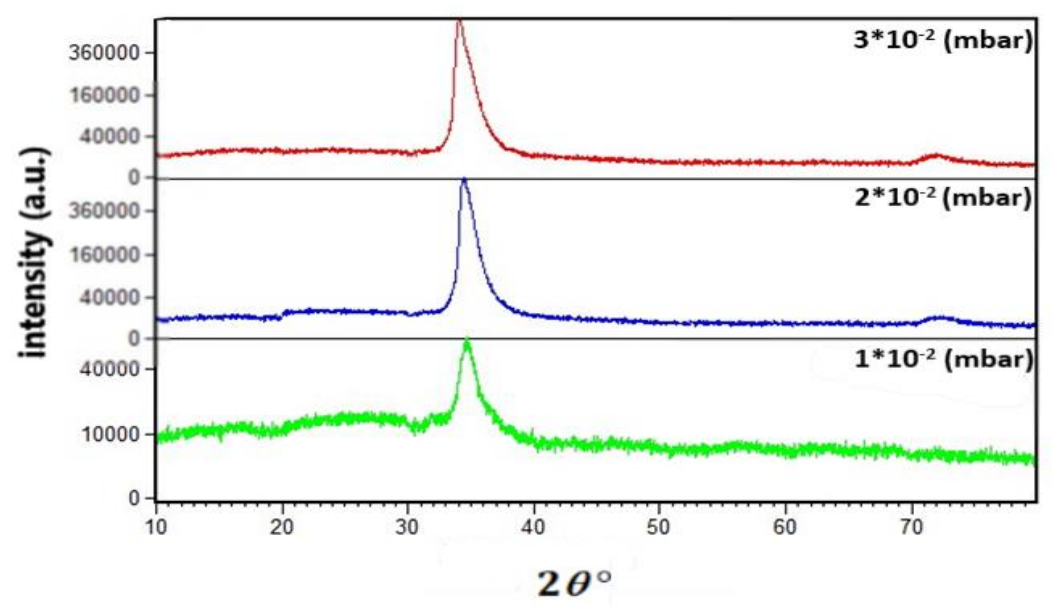

Fig1. Patterns of XRD for AZO films with changing of Ar gas pressure.

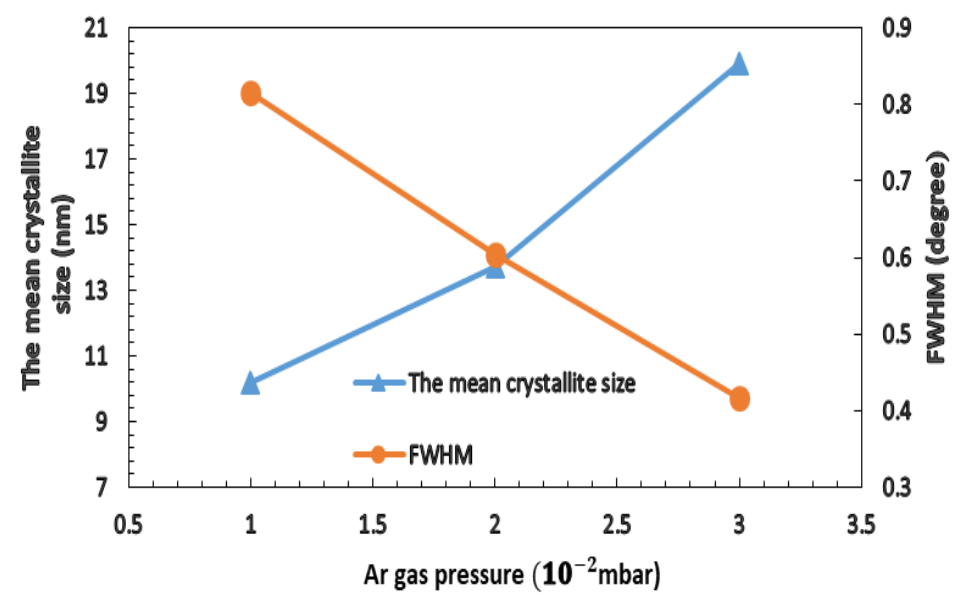

Fig2. The mean crystallite size and FWHM for AZO films with changing of Ar gas pressure from $1 \times 10^{-2} \mathrm{mbar}$ to $3 \times 10^{-2} \mathrm{mbar}$.

We can quantify the defects in the AZO thin films for the highest intensity peak (002) by computing the microstrain and dislocation density by below equations;

$\varepsilon=\frac{\beta \cot \theta}{4}$

$\delta=\frac{1}{D^{2}}$

Where $\varepsilon$ is the micro strain and $\delta$ is the dislocation density, the calculated results are low and with increasing Ar gas pressure are decreased which shows the presence of minimum defects in these films [7], the examined results are listed in table1. 
For calculating the number of crystallite $(\mathrm{N})$ per unit area, we can use below equation:

$N=\frac{t}{D^{3}}$

Where $\mathrm{t}$ is the thickness of films and $\mathrm{D}$ is the mean crystallite size, with improving Ar gas pressure the number of crystallites decreased that is against the variation of the mean crystallite size [7], the results are listed in table 1.

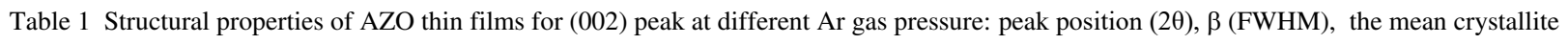
size (D), d-spacing (d), micro strain $(\varepsilon)$, dislocation density $(\delta)$, and number of crystallite $(\mathrm{N})$

\begin{tabular}{|c|c|c|c|c|c|c|c|}
\hline The XRD analysis & & & & & & & \\
\hline $\begin{array}{l}\text { Ar gas pressure } \\
\left(10^{-2} \text { mbar }\right)\end{array}$ & $\begin{array}{c}\text { peak position } \\
(2 \theta)\left({ }^{\circ}\right)\end{array}$ & $\beta\left(^{\circ}\right)$ & D (nm) & d $(\AA)$ & $\varepsilon$ & $\delta^{*} 10^{16}$ & $\mathrm{~N}^{* 10^{16}}$ \\
\hline 1 & 34.40 & 0.8160 & 10.2 & 2.6041 & 0.0114 & 0.961 & 34.3 \\
\hline 2 & 34.394 & 0.6048 & 13.7 & 2.6053 & 0.0084 & 0.532 & 17.1 \\
\hline 3 & 34.388 & 0.4171 & 19.9 & 2.6080 & 0.0058 & 0.252 & 7.2 \\
\hline
\end{tabular}

\section{3-1-2. The FESEM analysis}

Surface morphology plays an important role in investigating the change of different characteristics for any metal oxide thin films, in this paper, the FESEM study is used to realize the AZO film morphology, growth of grain and the estimation of nanoparticles size on surface of films with changing of Ar gas pressure [7]. Fig $3(\mathrm{a}-\mathrm{c}(200 \mathrm{~nm}))$ and $(\mathrm{d}-\mathrm{f}(1 \mu \mathrm{m}))$ indicate the investigation of FESEM images for various Ar gas pressure from $1 \times 10^{-2}$ mbar to $3 \times 10^{-2}$ mbar, as shown these images the mean approximate size of nanoparticles are increased with enhancing of Ar gas pressure from 35.98 to $72.93 \mathrm{~nm}$. 

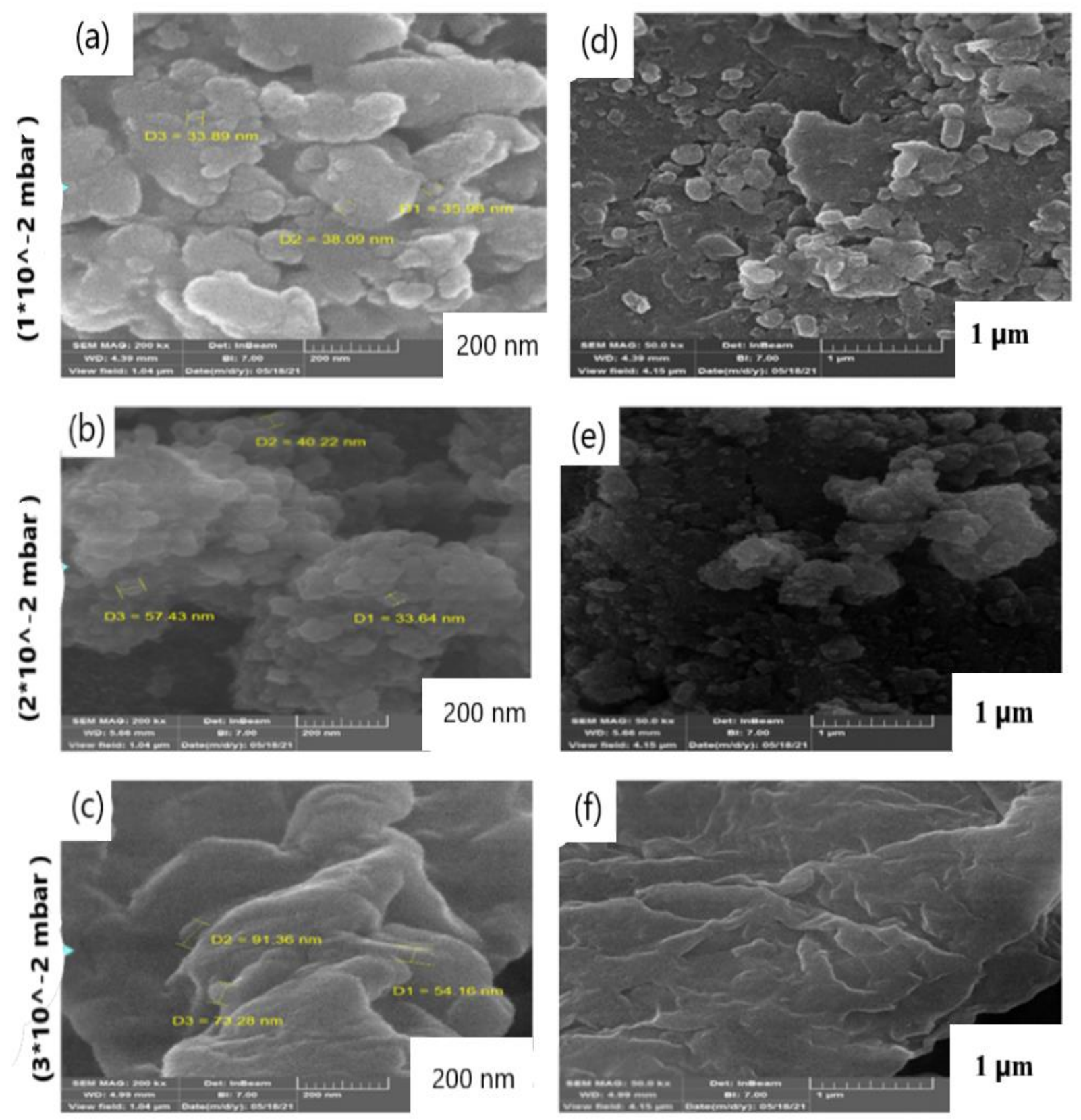

Fig 3. The FESEM images for AZO thin films with changing of Ar gas pressure from $1 \times 10^{-2}$ mbar to $3 \times 10^{-2}$ mbar.

\section{3-1-3. Atomic Force Microscopy (AFM)}

The 2-D and 3-D images of surface topography for the AZO thin films were studied by the AFM analysis as shown in Fig 4 .this analysis helps us to investigate the surface characteristics with numerical data. We know this tool converts surface data into illustrative images. Calculating the 
three-dimensional (3-D) surface data is crucial to control the quality of thin films to engineering the surface behavior for improved optical and electrical properties; any variation between peaks and valleys is more suitable for providing information about the 3-D surface topography. Experimental information extracted from the surface description of the AFM images (according to ISO 25178-2: 2012) for the three samples with different Ar gas pressure [32- 34]. The results of Root Mean Square (RMS) roughness parameter of films with Ar gas pressure from $1 \times$ $10^{-2} \mathrm{mbar}$ to $3 \times 10^{-2} \mathrm{mbar}$ are obtained to be 22.06 to $30.18 \mathrm{~nm}$, it is found that by increasing Ar gas pressure, the surface becomes rough as root-mean-square deviation $\left(S_{\mathrm{q}}\right)$, skewness of topography height distribution $\left(\mathrm{S}_{\mathrm{sk}}\right)$, kurtosis of topography height distribution $\left(\mathrm{S}_{\mathrm{ku}}\right)$ calculated from the AFM images are presented in Table 2. $\mathrm{S}_{\mathrm{z}}\left(S_{z}=S_{p}+S_{v}\right)$ shows the distance between the peak and the valley which has the highest value for AZO thin films with pressure of $2 \times$ $10^{-2}$ mbar so this result indicates this film has the most disorder.
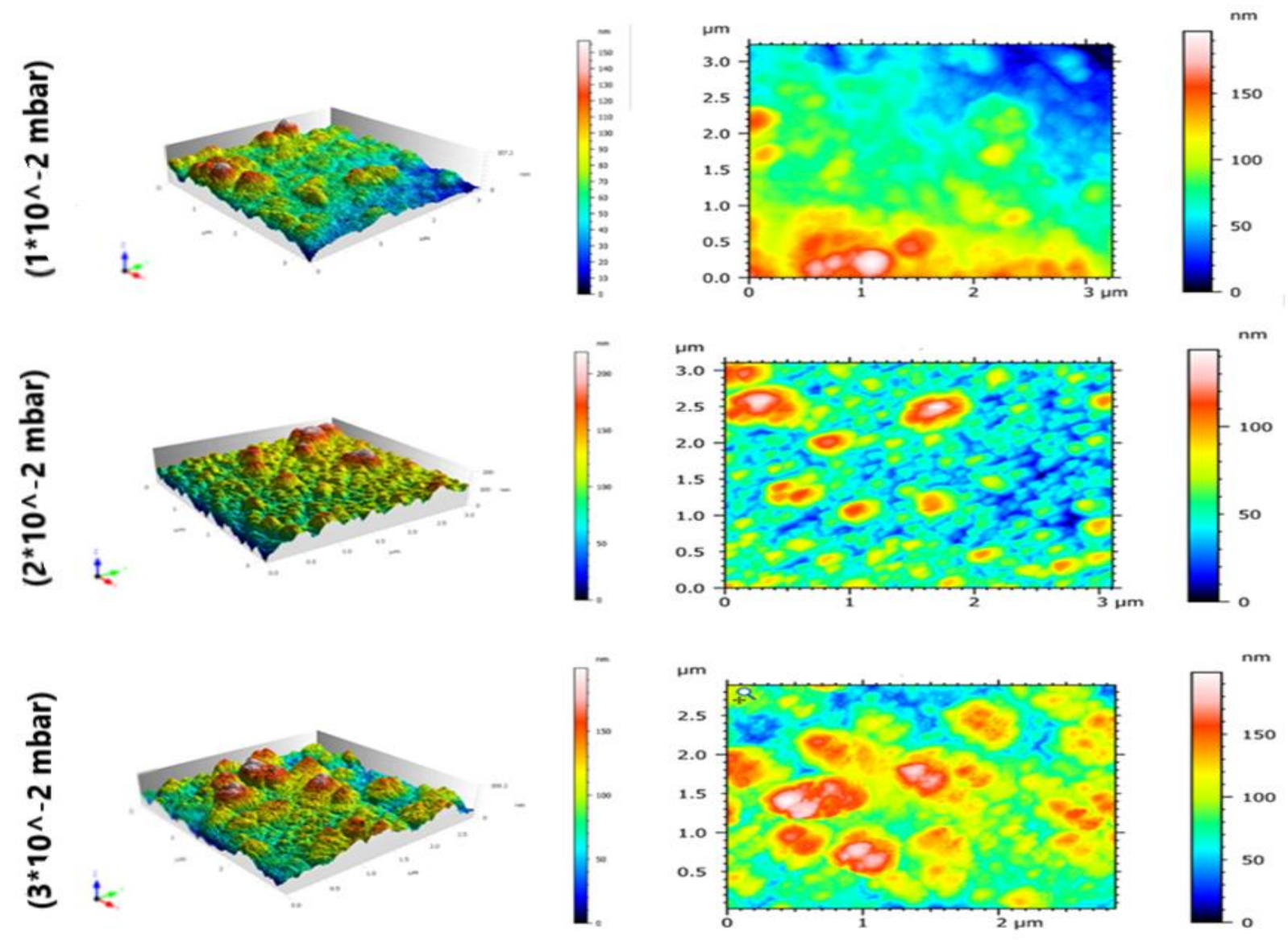

Fig 4. The 3-D and 2-D images of surface topography for AZO films at various Ar gas pressure

Table 2 The AFM information of AZO thin films 


\begin{tabular}{|c|c|c|c|c|c|c|c|}
\hline AFM analysis & & & & & & & \\
\hline $\begin{array}{c}\text { Ar gas } \\
\text { pressure } \\
\left(10^{-2} \text { mbar }\right)\end{array}$ & $\mathbf{S}_{\mathbf{q}}(\mathrm{nm})$ & $\mathbf{S}_{\text {sk }}$ & $\mathbf{S}_{\mathrm{ku}}$ & $\mathbf{S}_{\mathbf{p}}(\mathbf{n m})$ & $\mathbf{S}_{\mathbf{v}}(\mathbf{n m})$ & $\mathbf{S}_{\mathbf{z}}(\mathbf{n m})$ & $\mathbf{S}_{\mathbf{a}}(\mathbf{n m})$ \\
\hline 1 & 22.06 & 0.4563 & 4.064 & 92.46 & 64.78 & 157.2 & 16.33 \\
\hline 2 & 27.83 & 0.6032 & 4.324 & 107.8 & 111.2 & 219 & 20.63 \\
\hline 3 & 30.18 & 0.3925 & 2.955 & 101.6 & 97.63 & 199.2 & 24.02 \\
\hline
\end{tabular}

\section{3-2. Optical measurements}

\section{3-2-1. determination of optical band gap and the gap type (m)}

The optical transparency of film has an essential role in the characteristics of TCO thin films $[8,11,23,29$, 39]. In this work, the spectrums of optical transmittance were examined by UV-Visible spectroscopy in wavelengths range from 190 to $1100 \mathrm{~nm}$ for various Ar gas pressure. Fig 5 gives out the spectrum of average optical transmittance of the AZO films in the visible region by enhancing of Ar gas pressure from $1 \times$ $10^{-2}$ mbar to $3 \times 10^{-2}$ mbar decreases from $87.5 \%$ to 73 . $\%$, also the film with the least Ar gas pressure exhibit the maximum average transmittance of $87.5 \%$, the calculated parameters are listed in Table 3. Fig 6 shows the spectrum of absorption for different Ar gas pressure, it demonstrates the absorption decreases with enhancing wavelength with sharp absorption edge $[9,13,17,18]$.

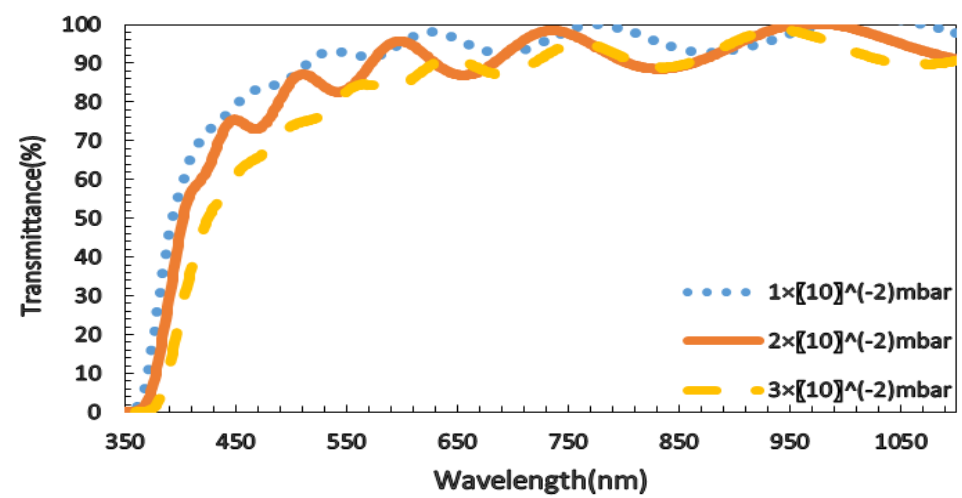

Fig5. The Optical transmittance of films with the Ar gas pressure changed from $1 \times 10^{-2} \mathrm{mbar}$ to $3 \times 10^{-2} \mathrm{mbar}$. 


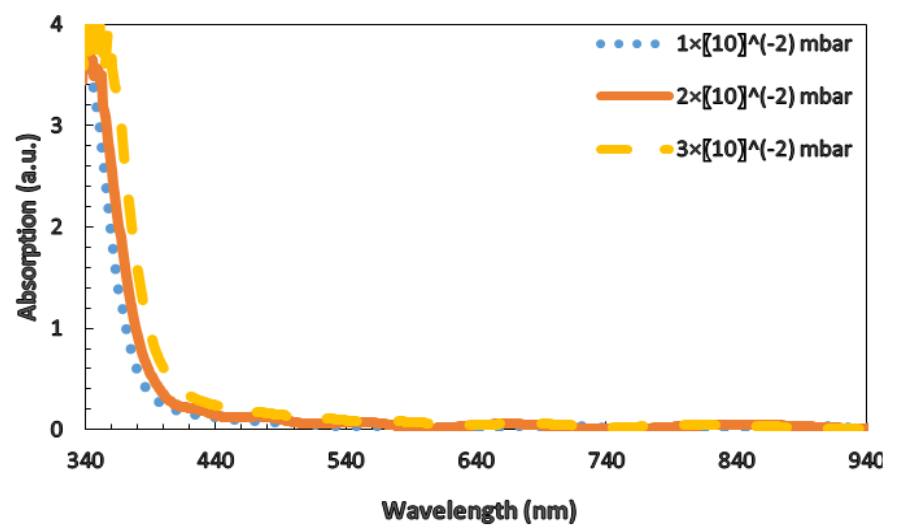

Fig6. The spectrum of Absorption for films with changing of the Ar gas pressure from $1 \times 10^{-2} \mathrm{mbar}$ to $3 \times 10^{-2} \mathrm{mbar}$.

In this present study, for calculating the band gap energy $\left(E_{g}\right)$, we use two technique, the ineffective thickness method (ITM) and Derivation of ineffective thickness method (DITM) in semiconductors which were presented with Eq 5 and Eq 8 respectively, for examining band gap we only need to examining the absorption instead of coefficient absorption, without requiring thickness of films, with following equations [39]:

$$
\begin{aligned}
& A(v) h v=K\left(h v-E_{\text {gap }}\right)^{m} \\
& \operatorname{Ln}(A(v) E)=\operatorname{Ln}\left(K\left(h v-E_{\text {gap }}\right)^{m}\right) \\
& \operatorname{Ln} A((v) E)=m \operatorname{Ln}(K)+m \operatorname{Ln}\left(E-E_{\text {gap }}\right)
\end{aligned}
$$

And derivation of $\mathrm{Eq}(7)$ is:

$\frac{d\{\operatorname{Ln}[A(v) E)\}}{d(E)}=\frac{m}{E-E_{g a p}}$

That $A(v)$ is the absorption and $\mathrm{K}$ is a constant, $\mathrm{m}$ is the transition index where values of $\mathrm{m}$ depend on the nature of the optical transition type that can have various measurements such as $m=1 / 2$ for allowed direct, $\mathrm{m}=3 / 2$ for forbidden direct, $\mathrm{m}=2$ for allowed indirect and $\mathrm{m}=3$ for forbidden indirect optical transition, respectively. If the graphs of $\frac{d\{L n[A(v) E)\}}{d(E)}$ versus $E$ are drawn, there would be a discontinuity at $E=E_{g a p}$. In this study, in DITM, the evaluated band gap energy for various Ar gas pressure are from 3.23 to $3.32 \mathrm{eV}$ , the increasing of band gap energy can be owing to a corresponding shift in the mean crystallite size which the mean crystallite size affects the optical characteristics obtaining higher band gap energy for thin films with smaller the mean crystallite size. Fig 7 and Fig 8 indicate the optical band gap for ITM and DITM respectively, where the values of optical band gap were reduced by enhancing Ar gas pressure [7, 8, 26, 38-40], Table 3 indicates the obtained results. 


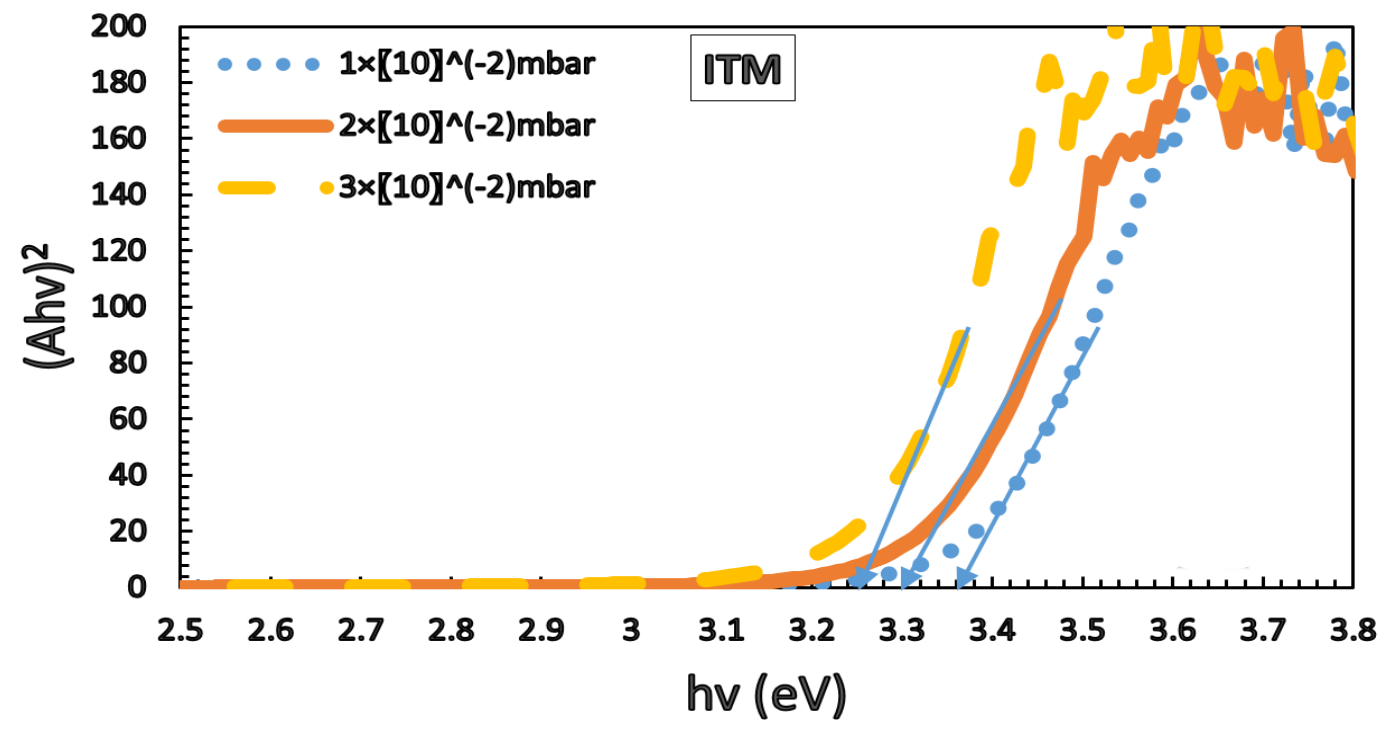

Fig7. The plots of $(A h v)^{2}$ vs hv at various Ar gas pressure from $1 \times 10^{-2}$ mbar to $3 \times 10^{-2}$ mbar.

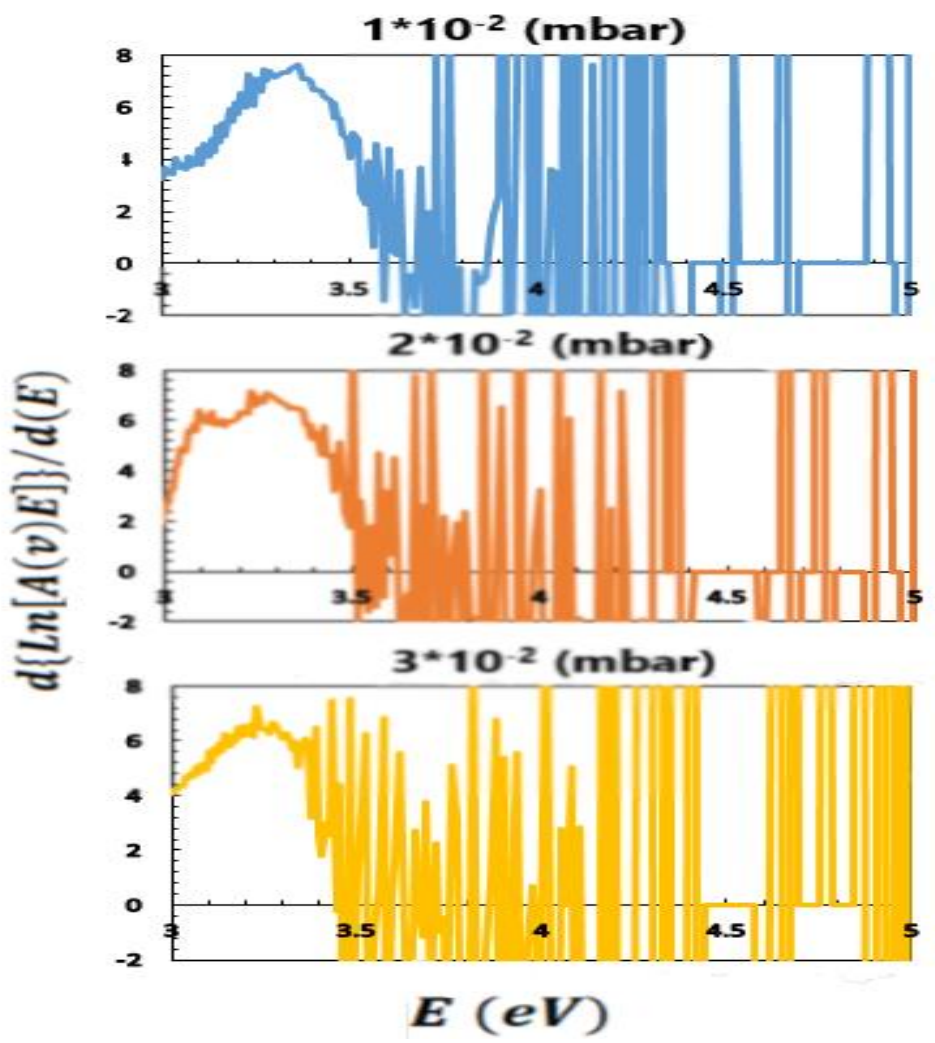

Fig8. The plots of $\frac{d\{L n[A(v) E]\}}{d(E)}$ versus hv at various Ar gas pressure. 
By utilizing the optical band gap energy values, examined from Fig 8, the transition index $(\mathrm{m})$ can be evaluated by the slope of the linear part of $\operatorname{Ln}(A E)$ versus $\operatorname{Ln}\left(E-E_{g a p}\right)$, the graphs were indicated in Fig 9. The calculated measurement are from 0.640 to 0.837 that they are almost about $\frac{1}{2}$ where shows direct allowed transition with changing of Ar gas pressure [21, 37, 39, 40], the calculated results listed in Table 3 .

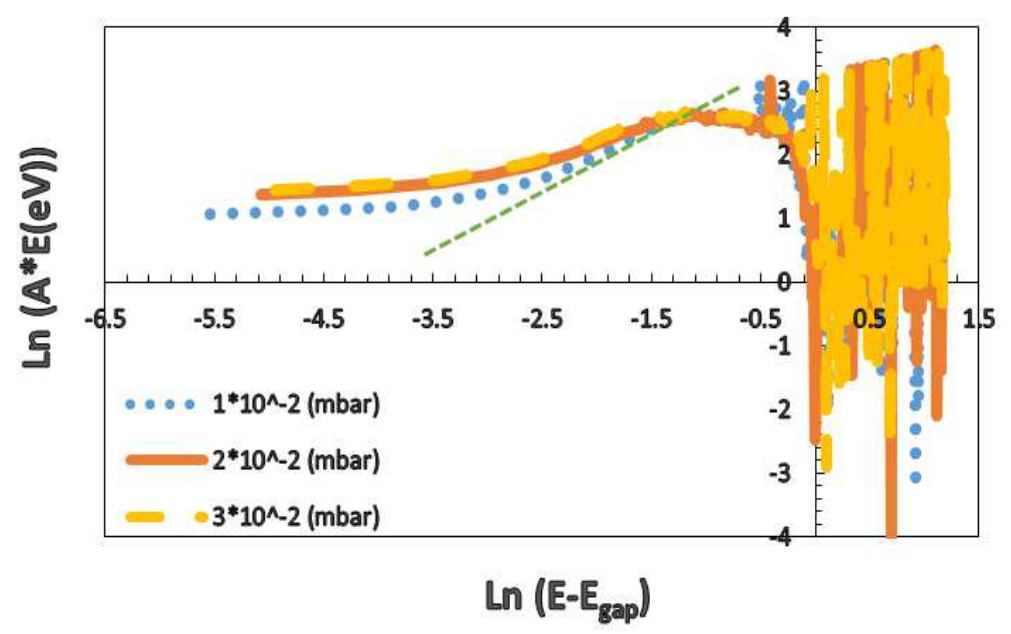

Fig9. The diagram of $\operatorname{Ln}(A E)$ versus $\operatorname{Ln}\left(E-E_{\text {gap }}\right)$, with changing of Ar gas pressure, a linear part was indicated for AZO films with Ar gas pressure of $1 \times 10^{-2}$ mbar.

\section{3-2-2 Urbach energy: examining the crystalline quality}

Urbach energy is the width of the tail of localized states as for the optical transition between localized tail states in the near to bands the Urbach tail can be obtained from the slope of straight plotting $\operatorname{Ln} A(v)$ versus the incident photon energy [36, 38, 39, 40-43]

$\operatorname{Ln} A(v)=\operatorname{Ln} A_{0}+h v / E_{\text {tail }}$

That $A_{0}$ is a constant, $\mathrm{h} v$ is the incident photon energy and $E_{\text {tail }}$ exhibits Urbach energy. Fig 10 shows the calculated of Urbach energy that the results are listed in Table 3 . The values of Urbach tail initially increased and then decreased with enhancing of Ar gas pressure are increased that decreasing of the Urbach tail can be considered a less number of defects where it causes the structural characteristics become more orderly The calculated values have been listed in Table 3 [37, 38, 40-43]. Fig 11 demonstrates the changing of band gap and Urbach tail as a function of Ar gas pressure that with enhancing of Ar gas pressure, the band gap is decreased and the Urbach tail initially increased and then decreased. 


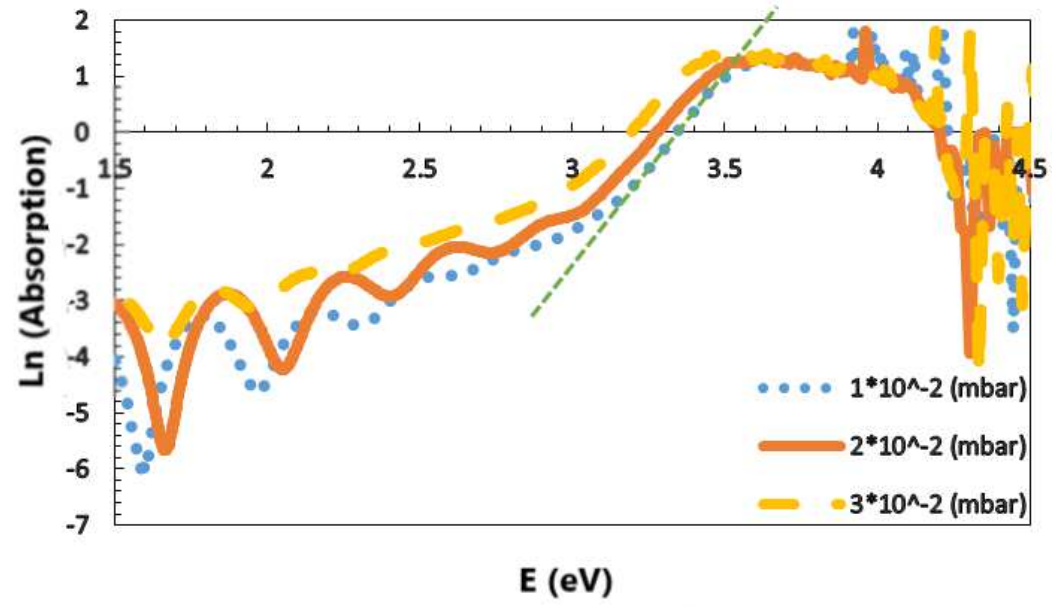

Fig10. The diagram of $\operatorname{Ln} A$ versus $\mathrm{E}$ with changing Ar gas pressure, for obtaining Urbach tail, a typical linear part was indicated for AZO thin film by Ar gas pressure of $1 \times 10^{-2}$ mbar.

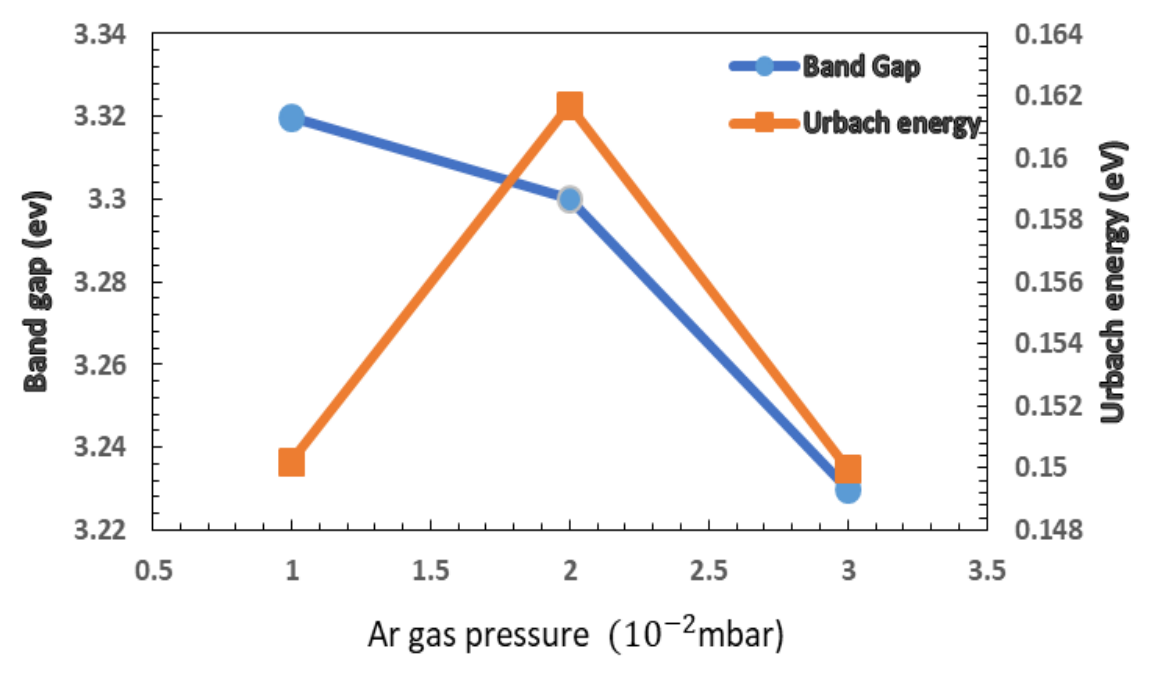

Fig11. The variation of band gap energy and Urbach energy with increasing Ar gas pressure from $1 \times$ $10^{-2} \mathrm{mbar}$ to $3 \times 10^{-2} \mathrm{mbar}$.

$\sigma$ is the steepness parameter that characterizing the broadening of the optical absorption edges owing to electron-phonon interaction, was examined by the following equation[44]:

$\sigma=\frac{k_{B} T}{E_{u}}$

Where $\mathrm{k}_{\mathrm{B}}$ is the Boltzman constant and $\mathrm{T}$ is the absolute temperature in Kelvin, therefor the measurements of the strength of the electron -phonon interaction can be determined by the following equation: 
$E_{e l-p h}=\frac{2}{3 \sigma}$

Fig 12 shows with increasing in the Urbach energy values, the steepness parameter and the strength of electron-phonon interaction decreases and increases respectively, the results indicate with increasing $\mathrm{Ar}$ gas pressure the localized states initially increase and then decrease which may be resulted either from an increase or decrease in the vacancies or dislocation defects of the AZO thin films [44]. The measurements values are listed in table 3.

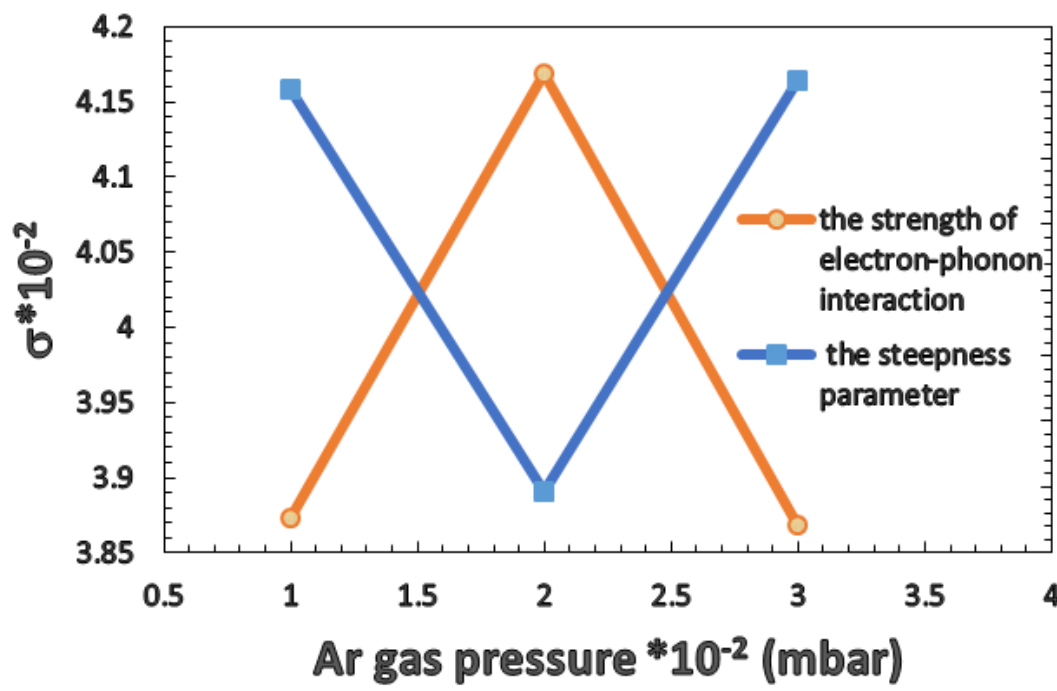

17.4

17.2

17

16.8

$16.6 \frac{\frac{5}{2}}{\frac{1}{6}}$

16.4

16.2

16

15.8

Fig12. The variation of the steepness parameter and the strength of electron-phonon interaction of AZO thin films at various Ar gas pressure.

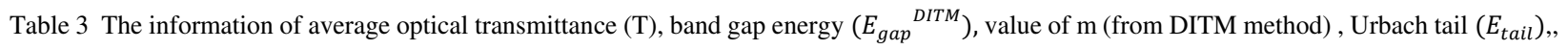
the steepness parameters $(\sigma)$, the strength of electron-phonon interaction $\left(E_{e l-p h}\right)$, for AZO films with changing of Ar gas pressure

\begin{tabular}{|c|c|c|c|c|c|c|}
\hline $\begin{array}{l}\text { Ar gas pressure } \\
\left(10^{-2} \text { mbar }\right)\end{array}$ & $\mathrm{T}(\%)$ & $E_{\text {gap }}{ }^{D I T M}(\mathrm{eV})$ & m (DITM) & $E_{\text {tail }}(\mathrm{eV})$ & $\sigma * 10^{-2}$ & $\overline{E_{e l-p h}}$ \\
\hline $1 \times 10^{-2} \mathrm{mbar}$ & 87.5 & 3.32 & $\begin{array}{l}0.837 ; \text { direct } \\
\text { allowed transition }\end{array}$ & 0.1502 & 17.211 & 3.873 \\
\hline $2 \times 10^{-2} \mathrm{mbar}$ & 79 & 3.30 & $\begin{array}{l}0.640 \text {; direct } \\
\text { allowed transition }\end{array}$ & 0.1617 & 15.987 & 4.169 \\
\hline $3 \times 10^{-2} \mathrm{mbar}$ & 73 & 3.23 & $\begin{array}{l}0.699 \text { direct } \\
\text { allowed transition }\end{array}$ & 0.1500 & 17.234 & 3.868 \\
\hline
\end{tabular}

\section{3-2-3 Determination of Refractive index and dielectric constant}

\section{3-2-3-1 Refractive index and dielectric constant in the visible region: determining dissipation factor and the lattice dielectric constant}


Some optical features such as Refractive index (n) and extinction coefficient (k) play an essential part for any TCO film. The refractive index (n) was examined from below equation:

$n=\frac{(1+R)}{(1-R)}+\sqrt{\frac{4 R}{(1-R)^{2}}-k^{2}}$

Where $\mathrm{R}$ is the reflectance and $\mathrm{k}$ is the extinction coefficient measurements, Fig 13 indicate the difference of the refractive index versus the wavelength where the spectral range of $n$ is reduces with enhancing wavelength because of the increasing of transmittance that may be because of light scattering. The average measurements of refractive index examined in the Visible region, they are in the range of 1.612 to 2.001 that the refractive index with enhancing of Ar gas pressure owing to the increasing of the scattering of photon light is increased $[7,38,45]$. The examined data are shown in table 4.

The extinction coefficient $(\mathrm{k})$ measurement can be calculated from the below equation:

$k=\frac{\alpha(\lambda) \lambda}{4 \pi}$

That $\alpha(\lambda)$ is the absorption coefficient of AZO film .Fig 14 indicates the variation of the extinction coefficient versus wavelength that the calculated results for various of Ar gas pressure is low for all wavelength range.it may be due to the improvement of crystalline structure, smooth surface, reducing of defects and the high transmittance of the sputtered AZO films [7, 38, 45], also the calculated measurements are listed in table4.

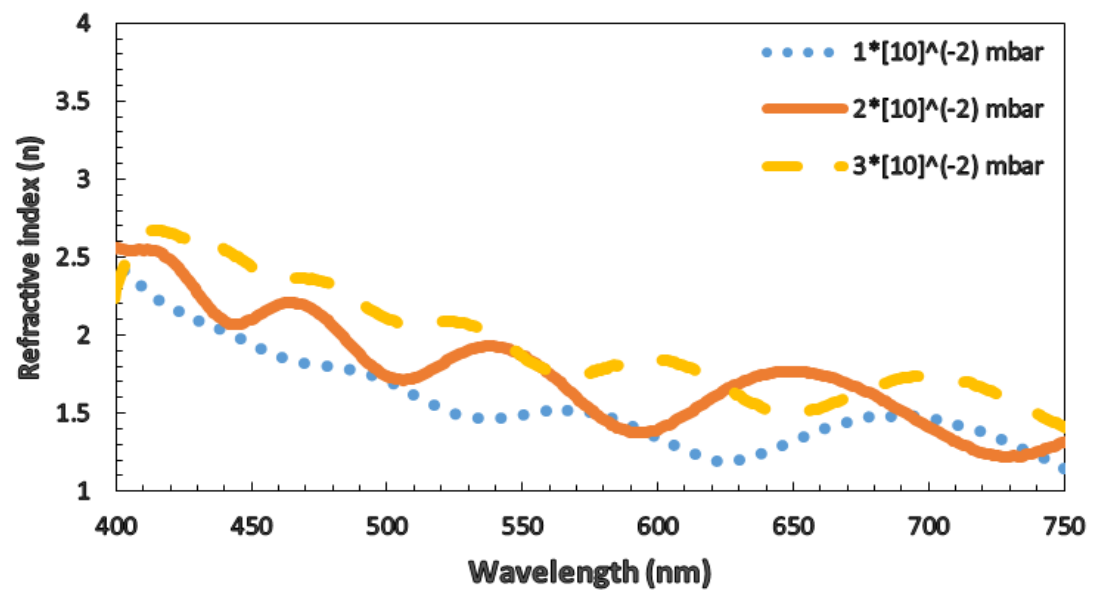

Fig 13. the difference of the refractive index vs the wavelength with increasing Ar gas pressure from $1 \times 10^{-2}$ mbar to $3 \times 10^{-2} \mathrm{mbar}_{\text {. }}$ 


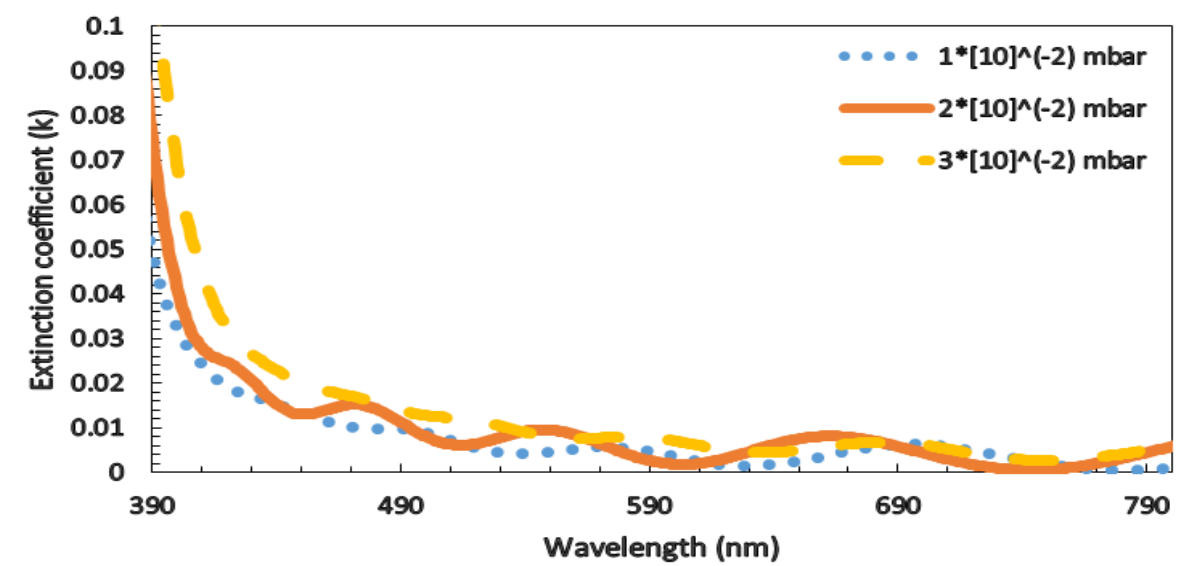

Fig 14 the variation of the extinction coefficient versus the wavelength with increasing Ar gas pressure from $1 \times 10^{-2} \mathrm{mbar}$ to $3 \times$ $10^{-2}$ mbar.

The complex dielectric constant $\varepsilon\left(=\varepsilon_{1}+i \varepsilon_{2}\right)$ can define the optical characteristics of any solid material that the dielectric feature such as real part $\left(\varepsilon_{1}\right)$ and imaginary part $\left(\varepsilon_{2}\right)$ of dielectric constants were calculated from the below equations:

$\varepsilon_{1}=n^{2}-k^{2}$

$\varepsilon_{2}=2 n k$

Figs 15 and 16 indicate the difference of the real and imaginary part of dielectric constants versus incident photon energy for AZO films by different Ar gas pressure. The dielectric constants measurement is most appropriate for TCO thin films that the calculated results for this study are low and the calculated real part $\left(\varepsilon_{1}\right)$ were higher than the imaginary part $\left(\varepsilon_{2}\right)$ measurements for these films [7, 38, 45]. the results summarized in Table 4.

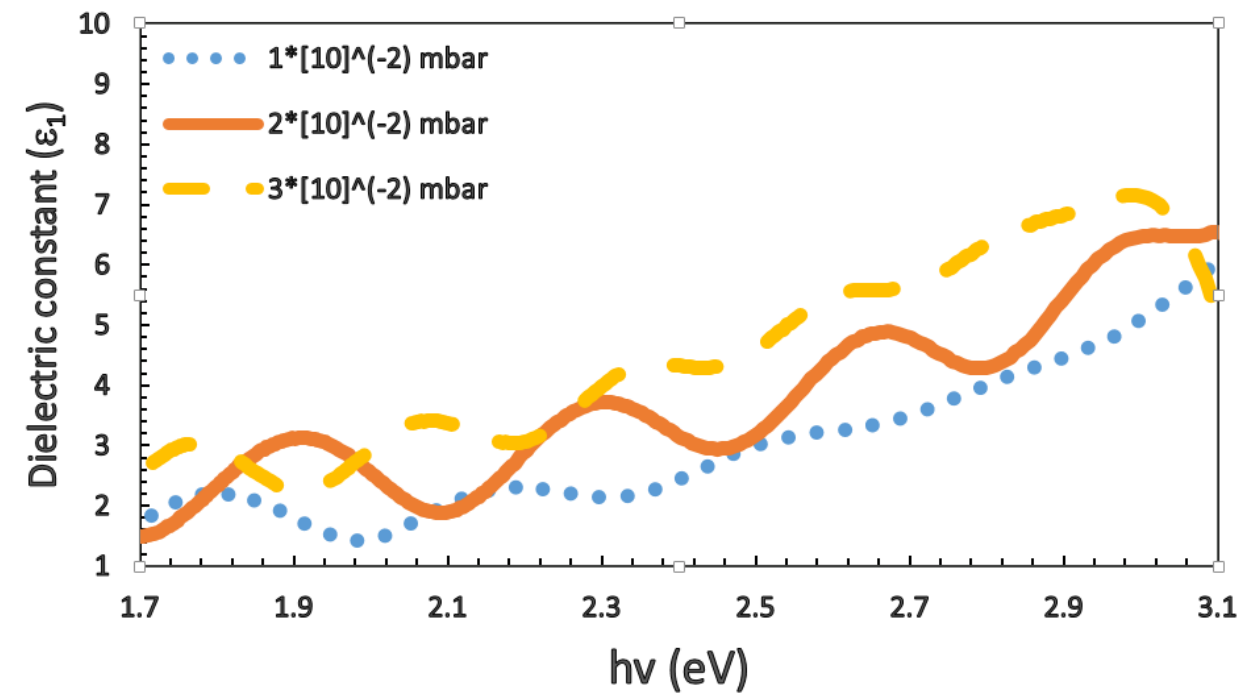

Fig 15 the variation of the real part of dielectric constant vs incident photon energy with increasing Ar gas pressure from $1 \times$ $10^{-2}$ mbar to $3 \times 10^{-2}$ mbar. 


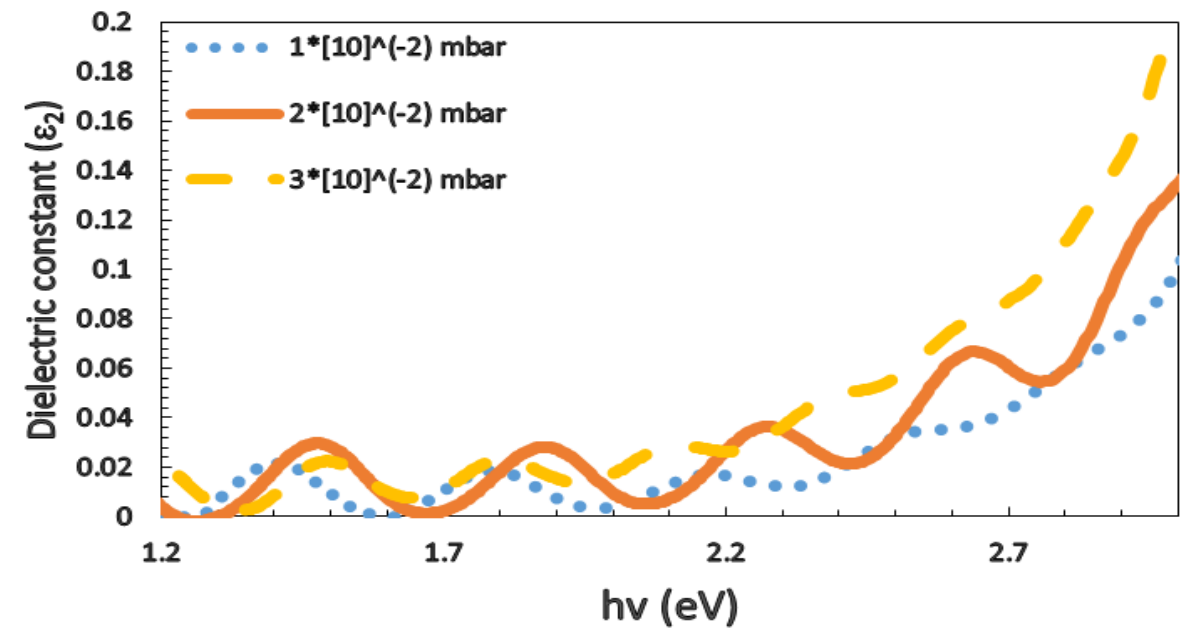

Fig 16 the variation of the imaginary part of dielectric constant vs incident photon energy with increasing Ar gas pressure from $1 \times 10^{-2} \mathrm{mbar}$ to $3 \times 10^{-2}$ mbar.

Dissipation factor $(\tan \delta)$ is an optical value which is dependent on real and imaginary part of dielectric constants, The measurements of dissipation factor of films determine by the below equation:

$$
\tan \delta=\frac{\varepsilon_{2}}{\varepsilon_{1}}
$$

Where $\varepsilon_{2}$ is the imaginary part of dielectric constant and $\varepsilon_{1}$ is the real part of dielectric constant, Fig 17 indicates the variation of $\tan \delta$ versus incident photon energy for AZO films with various Ar gas pressure that calculated results are low, it is can be due to the high optical transmittance and it minimizes the waste of electrical energy as heat which with increasing Ar gas pressure increases [7, 38, 45], the results listed in table 4.

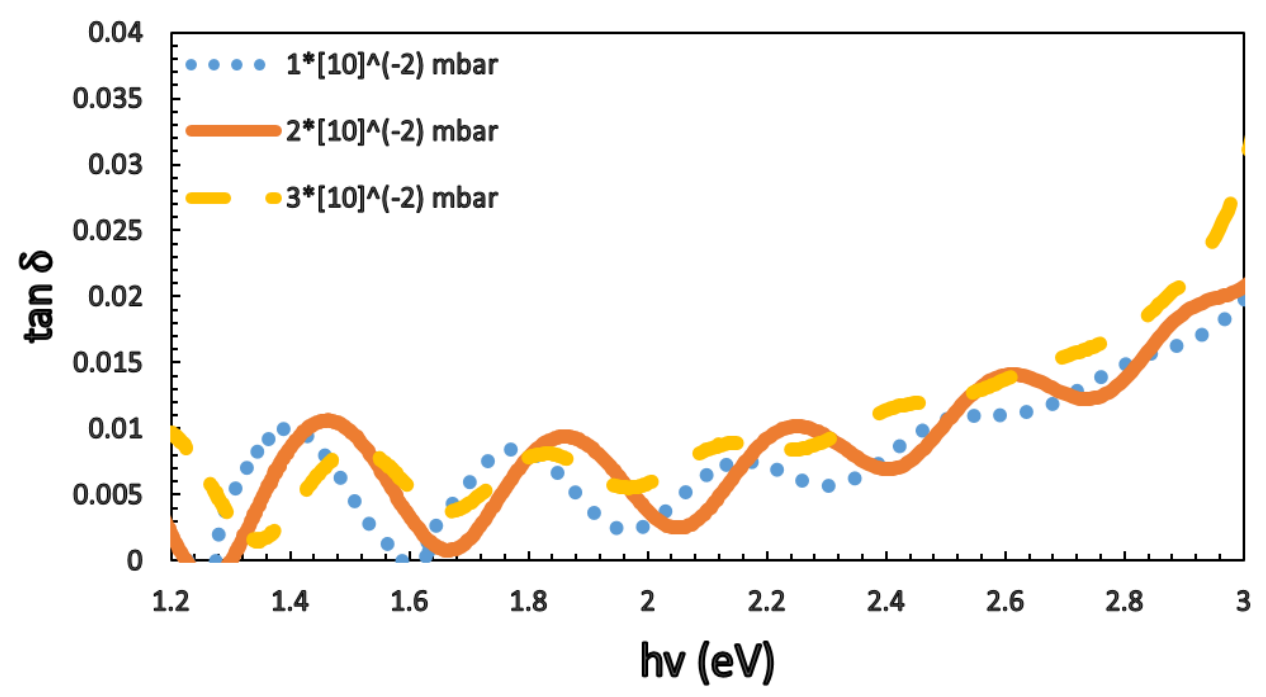


Fig 17 the variation of dissipation factor versus incident photon energy with increasing Ar gas pressure from $1 \times 10^{-2} \mathrm{mbar}$ to $3 \times 10^{-2} \mathrm{mbar}$.

The relation between the lattice dielectric constant $\left(\varepsilon_{L}\right)$ and the refractive index $\mathrm{n}$ can be described with the following equation:

$$
\varepsilon_{1}=n^{2}-k^{2}=\varepsilon_{L^{-}} \frac{e^{2} N}{4 \pi^{2} c^{2} \varepsilon_{0} m^{*}} \lambda^{2}
$$

Where $\varepsilon_{1}, \mathrm{e}, \mathrm{c}, \frac{N}{m^{*}}, \varepsilon_{\mathrm{L}}$ and $\varepsilon_{0}$ are the real part of dielectric constant ,the electron charge, the speed of light, the ratio of the free charge carrier concentration to the effective mass, the lattice dielectric constant and the permittivity of free space where the slope and vertical-axis intercept of the plots of $\varepsilon_{1}$ versus $\lambda^{2}$ results in $\frac{N}{m^{*}}$ and $\varepsilon_{\mathrm{L}}$, respectively (see Fig 18). the results of $\frac{N}{m^{*}}$ has the most value for AZO thin film with pressure of $3 \times 10^{-2}$ mbar localized state is decreased and it causes to transformation localized carrier concentration to the free charge carrier concentration also the values of $\varepsilon \mathrm{L}$ with enhancing of Ar gas pressure is increased, the results are listed in Table 4.[38,45]

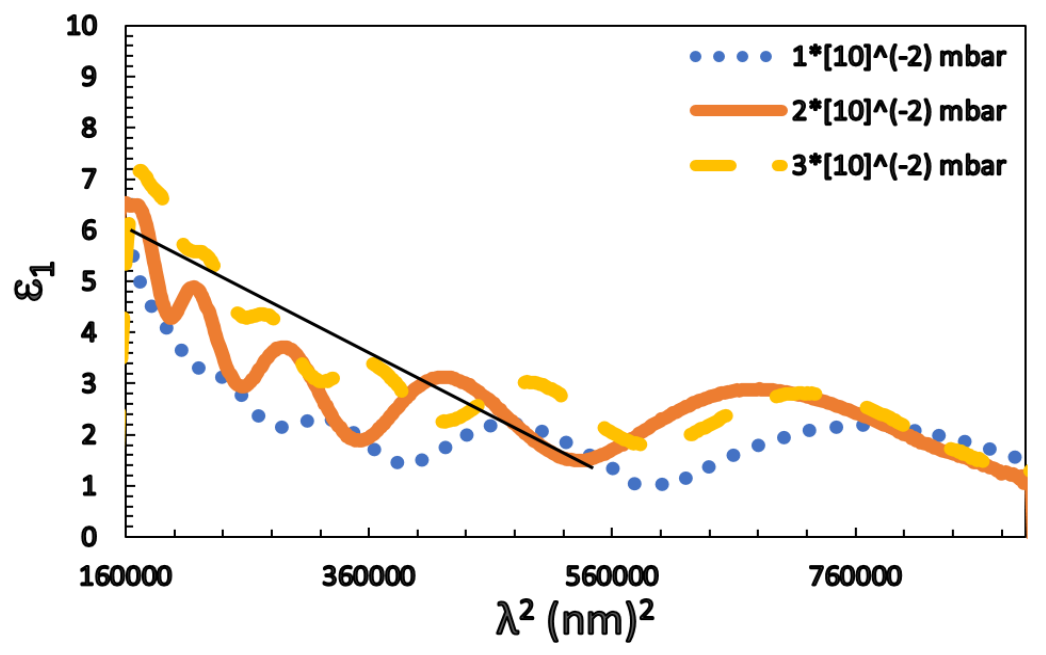

Fig 18. The variation of $\varepsilon_{1}$ versus $\lambda^{2}$ for AZO thin films at various $\mathrm{Ar}$ gas pressure.

\section{3-2-3-2 refractive index and dielectric constant at the absorption of edge}

To attain more intelligence into the optical ability of the thin films, one should try to measure some other optical parameter such as refractive index (n) and dielectric constant $(\varepsilon)$ at the absorption of edge that can be acquired using equation, introduced by Dimitrove and Sakaa [40 , 46, 47].

$\frac{n^{2}-1}{n^{2}+2}=1-\left(\frac{E g}{20}\right)^{\frac{1}{2}}$ 
$\varepsilon=n^{2}$

The calculated refractive index and the dielectric constant at different Ar gas pressure from $1 \times 10^{-2} \mathrm{mbar}$ to $3 \times 10^{-2}$ mbar is from 2.31 to 2.33 and 5.36 to 5.46 respectively that with increasing of Ar gas pressure are increased, the difference trends of these results and optical band gap are opposite, the results summarized in Table 4 [40,46,47].

Table 4 The information of the average of refractive index in the visible region (n), the average of extinction coefficient in the visible region (k), the real part of dielectric constant $\left(\varepsilon_{1}\right)$, the imaginary part of dielectric constant $\left(\varepsilon_{2}\right)$, dissipation factor $(\tan \delta)$, the ratio of the free charge carrier concentration to the effective mass $\left(\frac{N}{m^{*}}\right)$, the lattice dielectric constant $\left(\varepsilon_{L}\right)$, refractive index $\left(n_{\text {the edge of absorption }}\right)$ and dielectric constant $\left(\varepsilon_{\text {the edge of absorption }}\right)$, for AZO films with changing of Ar gas pressure.

\begin{tabular}{cllccccccc}
$\begin{array}{l}\text { Ar gas pressure } \\
\left(10^{-2} \text { mbar }\right)\end{array}$ & $n$ & $\mathrm{k}$ & $\varepsilon_{1}$ & $\varepsilon_{2}$ & $\tan \delta$ & $\frac{N}{m^{*}} *\left(10^{40} \mathrm{~m}^{-3} \mathrm{~kg}^{-1}\right)$ & $\varepsilon_{L}$ & $n_{\text {the edge of absorption }}$ & $\varepsilon_{\text {the edge of absorptior }}$ \\
\hline $1 \times 10^{-2}$ mbar & 1.612 & 0.0079 & 2.5984 & 0.025 & 0.0096 & 1.227 & 5.5366 & 2.31 & 5.36 \\
& & & & & & & & \\
$2 \times 10^{-2}$ mbar & 1.841 & 0.0100 & 3.3891 & 0.037 & 0.010 & 1.104 & & 2.32 & 5.38 \\
& & & & & & & 8.5195 & 2.33 & 5.46
\end{tabular}

\section{Conclusions}

- $\mathrm{Al}$ doped $\mathrm{ZnO}$ were prepared by DC-magnetorn sputtering with changing Ar gas pressure.

- The reaction of Ar gas pressure plays a main role in controlling the physical quantities of AZO films.

- There is no Al-related peaks were detected in the pattern of XRD, it is due to the aluminum was successfully incorporated into the $\mathrm{ZnO}$ lattice.

- The calculated results of micro strain and dislocation density show the presence of minimum defects in these films.

- The AFM analysis shows the RMS roughness parameter with enhancing of Ar gas pressure becomes rough and the distance between peaks and valley initially increases and then decreases that the AZO film with Ar gas pressure of $3 \times 10^{-2}$ mbar has the lowest disorder.

- The average spectrum of optical transmittance for AZO thin films with increasing of Ar gas pressure in the visible region was decreased, it is because of the increase in thickness of films.

- DITM method was reported to determine the band gap energy and transition index, the band gap energy with enhancing Ar gas pressure was reduced and the obtained results indicate AZO film is a semiconductor with the direct allowed transition. 
- The calculated results of Urbach tail are low it is because of the improvement of crystalline structure the deposited AZO thin film with pressure of $3 \times 10^{-2}$ mbar has the lowest Urbach tail and so the highest crystallinity nature.

- The calculated average values of the extinction coefficient for prepared films in the visible region are low and by increasing of Ar gas pressure is increased it is due to the surface of films become rough and the average optical transmittance is decreased .

- The calculated average values of dissipation factor are low, it is due to the high optical transmittance for films.

- The Refractive index and dielectric constant at the absorption of edge were increased with enhancing Ar gas pressure.

\section{Declaration}

Funding: Not applicable.

Conflicts of interest/Competing interests: The authors declare that they have no known competing financial interests or personal relationships that could have appeared to influence the work reported in this paper.

Availability of data and material: Not applicable.

Code availability: Not applicable.

Authors' contributions (optional: please review the submission guidelines from the journal whether statements are mandatory: Not applicable.

Ethics approval: Not applicable.

Consent to participate: Not applicable.

Consent for publication: Not applicable.

\section{Acknowledgements}

Not applicable.

\section{References}

[1] G.Torrisi, I.Crupi, S.Mirabella, A.Terrasi, Robustness and electrical reliability of AZO/Ag/AZO thin film after bending stress, Solar Energy Materials \& Solar Cells 165(2017)88-93.

[2] T.K.Subramanyam, P.Goutham, S.Pavan Kumar, S.R.Yadhuraj, K S.Geetha, Optimization of Sputtered AZO Thin Films for Device Application, Materials Today 5 (2018)10851-10859. 
[3] Sabah Habeeb Sabeeh, Ruaa Hashim Jassam, The effect of annealing and Al dopant on characterization of ZnO thin films prepared by sol-gel method, Results in Physics 10 (2018) 212-216.

[4] Malkeshkumar Patel, Joondong Kim, Electrical circuit of ITO/AZO/Ge photodetector, Data in Brief 14(2017)62-67.

[5] Bikash Sarma, Dipak Barman, Bimal K.Sarma, AZO (Al: ZnO) thin films with high figure of merit as stable indium free transparent conducting oxide, Applied Surface Science 479 (2019)786-795.

[6] Ju-Hyung Yun, Navaneethan Duraisamy, M.Melvin David Kumar, Joondong Kim, Optical and electrical of AZO/Ni/ITO transparent conductor, Materials Letters 143(2015)215-218.

[7] K.Deva Arun Kumar, S.Valanarasu, S.Rex Rosario, V.Ganesh, Mohd.Shkir, C.J.Sreelatha, S.AlFaify, Evaluation of the structural, optical, and electrical properties of AZO thin films prepared by chemical bath deposition for optoelectronics, Solid State Sciences 78 (2018) 58-68.

[8] Deok Kyu Kim, Hong Bae Kim, Room temperature deposition of Al- doped thin films on glass by RF magnetron sputtering under different Ar gas pressure, Journal of Alloys and Compounds 509 (2011) 421-425.

[9] K. Samanta, P. B. hattacharya, R. S. Katiyar, Raman scattering studies of p-type Sb doped ZnO thin films, J.Appl.Phys 108 (2010) 3501-3504.

[10] Long Wen, Bibhuti B.Sahu, Hye R.Kim, Jeon G.Han, Study on the electrical, optical, structural, and morphological properties of highly transparent and conductive AZO thin films prepared near room temperature, Applied Surface Science 473 (2019) 649-656.

[11] Yanping Xia, Peihong Wang, Gang He, Miao Zhang, Shiwei Shi, Yanmei Liu, Zhaoqi Sun, Microstructure, optoelectrical and pre-strain dependent electrical properties of AZO films on flexible glass substrates for flexible electronics, Surface \&Coatings Technology 320 (2017) 34-38.

[12] Shih-Feng Tseng, Investigation of post-annealing aluminum-doped zinc oxide (AZO) thin films by a graphene-based heater, Applied Surface Science 448 (2018) 163-167.

[13] Afrina Sharmin, Samia Tabassum, M.S.Bashar, Zahid Hasan Mahmood, Depositions and characterization of sol-gel processed Al-doped $\mathrm{ZnO}$ (AZO) as transparent conducting oxide(TCO) for solar cell application, Journal of Theoretical and Applied Physics 13 (2019)123-132.

[14] Mui Li, Lam, Azmizam Manie Mani, Saafie Salleh, Afishah Alias. Effect of Flexible Substrates on the Structural and Optical Properties of ZnO Films Deposited by Sputtering Method, Advanced Materials Research 11017 (2015) 678-683.

[15] A.Zaiour, A.Benhaya, T.Bentrcia, Impact of deposition methods and doping on structural, optical and electrical properties of ZnO-Al thin films, Optik-International Journal for Light and Electron Optics 186 (2019) 293-299.

[16] Umit Ozlem Akkaya Arier, Bengu Ozugur Uysal, Effects of precursor parameters on the optical and electrical properties of AZO nanocomposite films, Optic 127 (2016) 5065-5069.

[17] S.Rahmane, M.A.Djouadi, M.S.Aida, N.Barreau, B.Abdallah, N.Hadj Zoubir, Power and pressure effects upon magnetron sputtered aluminum doped ZnO films properties, Thin Solid Films, 519(2010)5-10. 
[18] Mrngying Wang, Qirong Liu, Guobo Dong, Yingchun He, Xungang Diao, Influence of thickness on the structure, electrical, optical and electrochromic properties of AZO thin films and their inorganic all-solid-state devices, Electrochimica Acta 258 (2017) 1336-1347.

[19].N.Evcimen Duygulu, A.O.Kodolbas, A.Ekerim Effects of argon pressure and r.f. power on magnetron sputtered aluminum doped ZnO thin films, Journal of Crystal Growth 394(2014)116-125.

[20] Saravanakumar M, Agilan S, Muthukumarasamy N, Effect of annealing temperature on characterization of ZnO thin films by sol-gel method, Int J Chem Tech Res:6(5) (2014) 2941-5.

[21] Tien-Chai Lin, , Wen-Chang Huang, Fu-Chun Tsai, The structural and electro-optical characteristics of AZO/Cr:Cu/AZO transparent conductive film, Thin Solid Film 589 (2015) 446-450.

[22] Juhnevica I, MASONKINA m, Mezinskis G, Gabrene A. Synthesis and properties ZnO /Al thin films prepared by dipcoating process, Mater Sci Chem 31(2015) 33-8.

[23] Ka Eun Lee, Mingsong Wang, Eui Jung Kim, Sung Hong Hahn, Structural and optical properties of sol-gel AZO thin film, Current Applied Physics 9 (2009) 683-687.

[24].Muhammad R.Islam, Mukhlasur Rahman, S.F.U.Farhad, J.Podder, Structural, optical, and phptocatalysis properties of solgel deposited Al-doped ZnO thin films, Surfaces and Interfaces 16 (2019)120-126.

[25] A.Valentini, F.Quaranta, M.Penza, F.R.Rizzi, The stability of zinc oxide electrodes fabricated by dual ion beam sputtering, J.Appl.Phys 73(1993)1143-1145.

[26]. Joydip Sengupta, R.K.Sahoo, C.D.Mukherjee, Effect of annealing on the structural, topographical and optical properties of sol-gel derived $\mathrm{ZnO}$ and AZO thin films, Materials Letters 83(2012) 84-87.

[27] M.A.Kaid, A.Ashour, Preparation of ZnO -doped Al films by spray pyrolysis technique, Appl. Surf. Sci 235(2007)30293033.

[28] T.Terasako, M.Yagi, M.Ishizaki, Y.Senda, H.Matsuura, S.Shirakata, Growth of ZnO films and nanowires by atmospheric pressure chemical vapor deposition using zinc powder and water as source materials, Surf .Coat.Technol.201(2007)8924-8930.

[29] Jingrong Chi, Ping Fan, Guangxing Liang, Dongping Zhang, Xingmin Cai, Zhuanghao Zheng and Tianbao Chen, ,Room

Temperature Deposition and Properties of AZO thin films by DC magnetron sputtering under different p;asma power, Advanced Materials Research, 194-196(2011)2440-2443.

[30] Guojia Fang, Dejie Li, Bao-Lun Yao,Fabrication and vacuum annealing of transparent conductive AZO thin films prepared by DC magnetron sputtering, Vacuum, 68(2002)363-372.

[31] G.J.Fang, D.J.Li, B.L.Yao ,Effect of vacuum annealing on the properties of transparent conductive AZO thin films prepared by DC magnetomagnetronring, Physica status solid, 193(2002)139-152.

[32] Ştefan Ţălu, Carlos Luna, Azin Ahmadpourian, Amine Achour, Ali Arman, Sirvan Naderi, Nader Ghobadi, Sebastian Stach, Behroz Safibonab. Micromorphology and fractal analysis of nickel-carbon composite thin films. Journal of Materials Science: Materials in Electronics November 2016, Volume 27, Issue 11, 11425-1143. 
[33] M. Molamohammadi, A. Arman, A. Achour and et al., Microstructure and optical properties of cobalt-carbon nanocomposites prepared by RF-sputtering J. Mater. Sci.: Mater. Electron. 26 (2015)5964-5969 .

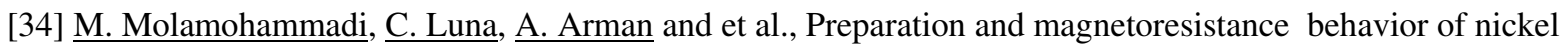
nanoparticles embedded in hydrogenated carbon filmJ. Mater. Sci.: Mater. Electron. 26 (2015) 6814-6818 .

[35] Mujdat Caglar, The effects of $\mathrm{Al}$ doping on the optical constants of $\mathrm{ZnO}$ thin films prepared by spray pyrolysis method, Journal of Materials Science: Materials in Electronics 19 (2008) 704-708.

[36] Aliasghar Shokri, Laya Dejam, Experimental and theoretical investigation on temperature and voltage dependence of Au/AZO thin-film Schottky diode'; International Nano Letters 9 (2019)161-168.

[37] Weihai Zhang, Juan Xiong, Lei Liu, Xianghui Zhang, Haoshuang Gu, Influence of annealing temperature on structural, optical and electrical properties of AZO/Pd/AZO films, Solar Energy Materials \& Solar Cells 153(2016)52-60.

[38] Laya Dejam, Shahram Solaymani, Amine Achour, Sebastian Stach, Stefan Talu, Negin Beryani Nezafat, Vali Dalouji, Ali Asghar Shokri, Atefeh Ghaderi, Correlation between surface topography, optical band gaps and crystalline properties of engineered AZO and CAZO thin films, Chemical Physics Letters 719 (2019)78-90.

[39] Nader Ghobadi, Derivation of ineffective thickness method for investigation of the exact behavior of the optical transitions in nanostructured thin films, J Master Sci: Master Electron 27 (2016) 8951-8956.

[40] N Ghobadi M Shiravand and E Gholami Hatam, Influence of sputtered time on the structural and optical characterization of Al-doped ZnO thin films prepared by RF sputtering technique, Optical and Quantum Electronics 53 (2021)14.

[41] Okba Belahssen, Said Benramache, Boubaker Benhaoua, Effect of Urbach energy with precursor molarity on the crystallite size in undoped ZnO thin film, Main Group Chemistry 13 (2014) 343-352.

[42] Urbach Franz, The long-wavelength edge of photographic sensivity and of the electronic absorption of solids, Physical Review 92 (1953) 1324.

[43] A.S.Hssanien, Alaa A.Akl, Effect of Se addition on optical and electrical properties of chalcogenide CdSSe thin films, Superlattices and Microstructures 89(2016)153-169.

[44] M .S .Bashar, R. Matin, M. Sultana, A. Siddika, M. Rahaman, M. A Gafur,F. Ahmed; Effect of rapid thermal annealing on structural and optical properties of $\mathrm{ZnO}$ thin films fabricated by RF magnetron sputtering technique, Journal of Theoretical and Applied Phys 14,(2020)53-68.

[45] A Qassim Abdullah, Surface and volume energy loss ,optical conductivity of Rhodamine 6G dye (R6G), Chemistry and Materials Reserch, 3 (2013)56-63.

[46] Dimitrov.V, Sakaa.S, Electronic oxide polarizability and optical basicity of simple oxidesI. Journal of Applied Physics, 79 (1996)1736-1740.

[47] Dimitrov.V, Sakaa.S, Linear and nonlinear optical properties of simple oxide.II, Journal of Applied Physics 79 (1996)17411745. 